\title{
Incidence of intussusception in children less than five years of age: a pre-rotavirus vaccine survey from Iran, 2010-2015
}

\author{
Abdoulreza Esteghamati ${ }^{1}$, Mohammadamin Joulani ${ }^{2}$, Shirin Sayyahfar*1미, Sarvenaz Salahi ${ }^{3}$, Mahla Babaie ${ }^{4}$, \\ Ahmad Reza Shamshiri ${ }^{5}$, Alireza Fahimzad ${ }^{6}$
}

Received: 4 Sep 2019

Published: 29 Apr 2020

\begin{abstract}
Background: The aim of this study was to determine the baseline statistics of intussusception in the under-five- year age group in Iran to facilitate the monitoring of potential side effects after administration of rotavirus vaccine.

Methods: This hospital-based historical cohort study reviewed children under 60 months of age with the final diagnosis of intussusception, ICD-10 code K56.1, using census in all hospitals of Tehran, Iran from March 2010-2015. Demographic (sex, age, hospital stay duration), clinical manifestations (such as currant jelly stool, abdominal pain, vomiting, and fever),diagnostic and treatment methods (contrast enema, ultrasonography, laparotomy, and laparoscopy), and outcome data of patients aged less than 5 years with the diagnosis of intussusception were collected and analyzed using SPSS Version 16.0 (SPSS Inc., Chicago, IL, USA)

Results: In this study, 759 patients were diagnosed with intussusception; 309 (40.7\%) cases were less than 12 months old. The annual incidence of intussusception was 66.54 cases per 100.000 in children less than one-year-old and 31.61 cases per 100.000 in children less than five years old. The most common symptoms and signs were abdominal pain/irritability (94.2\%) and tenderness $(24.2 \%)$, respectively. The diagnostic method was ultrasound in $75.9 \%$ of cases. The most frequent anatomic location was the ileocolic region $(87.87 \%)$ and the most common treatment method was barium enema.

Conclusion: This research has provided a baseline statistic for childhood intussusception in Tehran prior to the administration of the rotavirus vaccine to provide a better comparison with post-introduction data.
\end{abstract}

Keywords: Incidence, Intussusception, Invagination, Pediatrics, Rotavirus vaccine

Conflicts of Interest: None declared

Funding: Student Research Committee, Iran University of Medical Sciences (grant number: 97-01-193-33226).

\section{*This work has been published under CC BY-NC-SA 1.0 license. \\ Copyright $\odot$ Iran University of Medical Sciences}

Cite this article as: Esteghamati A, Joulani M, Sayyahfar Sh, Salahi S, Babaie M, Shamshiri AR, Fahimzad A. Incidence of intussusception in children less than five years of age: a pre-rotavirus vaccine survey from Iran, 2010-2015. Med J Islam Repub Iran. 2020 (29 Apr);34:40. https://doi.org/10.47176/mjiri.34.40

\section{Introduction}

Intussusception (invagination) is the most common cause of acute intestinal obstruction between 3 months and 3 years of age $(1,2)$. It is one of the most prevalent

Corresponding author:Dr Shirin Sayyahfar, sayyahfar.sh@iums.ac.ir

1. Research Center of Pediatric Infectious Diseases, Institute of Immunology and Infectious Diseases, Iran University of Medical Sciences, Tehran, Iran

2. Student Research Committee (SRC), School of Medicine, Iran University of Medical Sciences, Tehran, Iran

3. Minimally Invasive Surgery Research Center, Iran University of Medical Sciences, Tehran, Iran

4. Faculty of Medicine, Iran University of Medical Sciences, Tehran, Iran

5. Department of Epidemiology and Biostatistics, School of Public Health, Tehran University of Medical Sciences, Tehran, Iran

6. Pediatric Infections Research Center, Research Institute for Children Health, Shahid Beheshti University of Medical Sciences, Tehran, Iran pediatric intestinal and surgical emergencies in infants and young children (3) with a peak age of 4-10 months (1). While accurate data is not available in many developing

\section{$\uparrow$ What is "already known" in this topic:}

Intussusception is the most common cause of acute intestinal obstruction between 3 months and 3 years. Intussusception can be associated with some types of rotavirus vaccines such as RotaShield. Rotavirus vaccine is being planned to be included in Iran's national immunization program. Intussusception, associated with rotavirus vaccination, is a potential safety concern.

\section{$\rightarrow$ What this article adds:}

The annual incidence of intussusception in the pre-vaccine set up was 66.54 cases per 100.000 in children less than one-yearold and 31.61 cases per 100.000 in children less than five years old. This study is expected to be used in risk/benefit analysis of the rotavirus vaccination program in Iran in the future. 
countries, the incidence of intussusception in infants less than one year of age is between 34 and 100 per 100,000 children in developed countries (4).

Although the etiology of intussusception in children still remains unknown, it has been proposed that intestinal invagination could be triggered by post-infection lymphoid hyperplasia of the gastrointestinal tract wall $(5,6)$.

A number of studies have found an association between infection with some viral and bacterial agents and intussusception such as adenovirus, rotavirus, Yersinia enterocolitica, and Campylobacter jejuni $(5,7)$. It is also rarely associated with some generations of rotavirus vaccine administration in some settings $(4,8)$.

Intussusception can be diagnosed by ultrasonography, contrast enema [hydrostatic, barium, or pneumatic based] or during operation; however, barium enema is considered the gold standard in the diagnosis of this disease $(2,9)$. Treatments include non-operative and operative reduction. The non-operative reduction can be performed through hydrostatic, barium or pneumatic based enema. The standard treatment is pneumatic reduction which is more preferred because of lower morbidity rate and over $84 \%$ success rate if implemented well-timed. The operative reduction is performed for the cases of unsuccessful nonoperative reduction or attendant with contra-indications like peritonitis, profound shock, perforation, or sepsis which can be done with or without resection $(2,9,10)$.

Since 1999, attention has been drawn to the intussusception because of its association with the first licensed rotavirus vaccine, RotaShield (Wyeth Laboratories Inc., Marietta, Pennsylvania) [rhesus rotavirus tetravalent vaccine (RRV-TV)]. RotaShield was removed from marketing following reports of an increased rate of intussusception after vaccine administration (11).

This association resulted in the renewal of the first vaccine and led to the introduction of two new vaccines, a pentavalent human-bovine reassortant vaccine [RV5; RotaTeq, Merck, West Point, Pennsylvania], and a monovalent human rotavirus vaccine (RV1; Rotarix, GlaxoSmithKline Biologics, Research Triangle Park, North Carolina). No increased rate of intussusception was documented in the United States for either vaccine (12).

Iran is one of the countries that have not yet included active immunization against rotavirus infection in the expanded program of immunization (EPI). But based on a cost -effectiveness study, introduction of rotavirus vaccine to the national immunization program (NIP) of Iran is highly beneficial in reduction of pediatric morbidity and mortality (13). According to the study of Mousavi Jarrahi et al, vaccinating cohort of 1231735 infants in Iran with 2 doses of Rotarix would prevent 32092 hospitalizations, 158750 outpatient visits, and 1591 deaths during the 5 year follow-up and there would be $\$ 15,192,568$ saving for the society (13).

According to 2009 WHO recommendation, the health policy of Iran is considering this vaccine in the NIP in the near future; so, documentation of the intussusception rate prior to the vaccine introduction and serial surveillance afterward is important and mandatory (14).

This study was designed to estimate the annual and five- year incidences of intussusception in the pre-vaccine set up with the aim of comparing these results with postvaccination data and assessing the possible trends in yearly incidence of intussusception. This study is expected to be used in risk/benefit analysis of rotavirus vaccination program in Iran in the future. Moreover, this study describes the characteristics of intussusception among Iranian children under 5 years old.

\section{Methods \\ Study Design}

This hospital-based historical cohort study reviewed children under 60 months of age diagnosed with intussusception, based on the international statistical classification of diseases and health related problems version10 (ICD10) code K56.1 from March 2010 to March 2015 in Tehran, Iran. From 146 hospitals [including teaching, governmental, private, charity and military hospitals], 80 hospitals had pediatric wards of which 16 hospitals had reportable cases. The remaining 64 hospitals, did not have facilities for diagnosis and/or treatment of invagination (e.g. surgery ward, pediatric surgeon, emergent ultrasonography or barium enema) so they referred their cases to the other 16 hospitals. One private hospital refused to provide its data. This study was conducted after receiving approval from the ethics committee of Iran University of Medical Sciences (Ethics code: IR.IUMS.REC 1395.9221216224).

\section{Case identification, data collection}

The process of case finding was started by using ICD10 code K56.1 in the archive of the hospitals. The files archived as "intussusception cases" were selected. Then the content of the files was checked and evaluated carefully to ensure the diagnosis was based on the Brighton Collaboration Intussusception Working Group definition (15). If the compatibility was at the levels of the diagnostic certainty, the case was included. Intussusception diagnosis was confirmed when there was an operative or autopsy record with or without pathologic documentation or imaging manifestations by ultrasonography or air/liquid enema compatible with the intussusception diagnosis which was approved by operative or non-operative treatment. The exclusion criterion was living outside of Tehran. It is important to point out that during the study period, no type of Rotavirus vaccine was made available to the general public in Iran.

After literature review in the recent past 10 years and considering the variables required for the future comparison of the pre- and post- rotavirus vaccination area survey, we designed our checklist. Then we collected the related demographic data and clinical characteristics including age, sex, admission date, hospital stay duration, signs and symptoms, discharge logs, medical procedures (surgical and non-surgical intervention), surgery reports, ultrasonography (US) and radiology reports and pathology documents, if available. To minimize the risk of bias and missing data, we rechecked all data and checklists by a second group and then entered the data into an Excel database. 
Since Tehran has several pediatric specialty centers, and there is a high probability of referral or dispatch of patients with the diagnosis of intussusception from nonspecialized centers to specialized centers, non-duplication of cases in the study was confirmed by the second team. This point was also taken into account in the sample collection and, analysis.

\section{Statistical methods}

For calculating annual incidence, we needed the total number of children less than 12 months of age and under 5 years old living in Tehran for each year from March 2010 to March 2015. This data was accessible from the census data collected by the Statistical Center and Infection Control Department of Iran Ministry of Health (16). To analyze demographic data and clinical characteristics, descriptive statistics were performed. The frequency of categorical variables including signs and symptoms, diagnosing method, treatment and the hospital stay time were reported as numbers and percentages. All statistical analyses were conducted by SPSS Version 16.0 (SPSS Inc., Chicago, IL, USA).

\section{Results}

During this surveillance, 759 confirmed cases of intussusception were detected ( 7 cases were excluded due to living out of Tehran), of which $525(69.2 \%)$ cases were male. In this study, 20 patients had recurrent intussusception from whom one patient had two and the remaining 19 cases had one episode of recurrence. The male-female ratio was 2.24:1 (2.38:1 and1.51:1 in $\leq 3$ and >3years, respectively).
The age of the cases ranged from one month to 5 years of which $309(40.71 \%)$ patients were less than one year old. The median, mean and mode of age were 16.00 , $19.63 \pm 13.21$ and 9 months, respectively. The Inter Quartile Range (IQR) was 18.00 months. 25th and 75th percentiles were 9.00 and 27.00 respectively. The majority of cases were below 10 months of age $(32.4 \%)$ with the peak incidence between 9 and 10 months (12.5\%). None of the patients had received any form of rotavirus vaccines.

Graph 1 demonstrates the frequency of under one-yearold children (divided bimonthly) in five incidence years from March 2010 to March 2015. Totally, 123 (16.2\%) cases were admitted in 2010, $163(21.5 \%)$ in 2011, $199(26.2 \%)$ in $2012,116(15.3 \%)$ in 2013 and $158(20.8 \%)$ in 2014 .

The annual incidence of intussusception for the total 5 years was 66.54 cases per 100, 000 in children less than one- year- old and 31.61 cases per 100,000 in children less than five years old.

Graph 2 reveals the trend line of intussusception incidence in under-one-year and under-5-year age groups during five years.

The highest frequency of the disease was in the spring $(32.3 \%)$ and secondly in autumn $(26.6 \%)$ in comparison to other seasons.

The most common symptom of intussusception was abdominal pain/irritability $(94.2 \%)$ and the most frequent sign was tenderness $(24.2 \%)$. Other recorded signs and symptoms are shown in the Table 1.

The classic triad of vomiting, passage of blood through the rectum and abdominal pain was detected in 104 $(13.7 \%)$ cases.

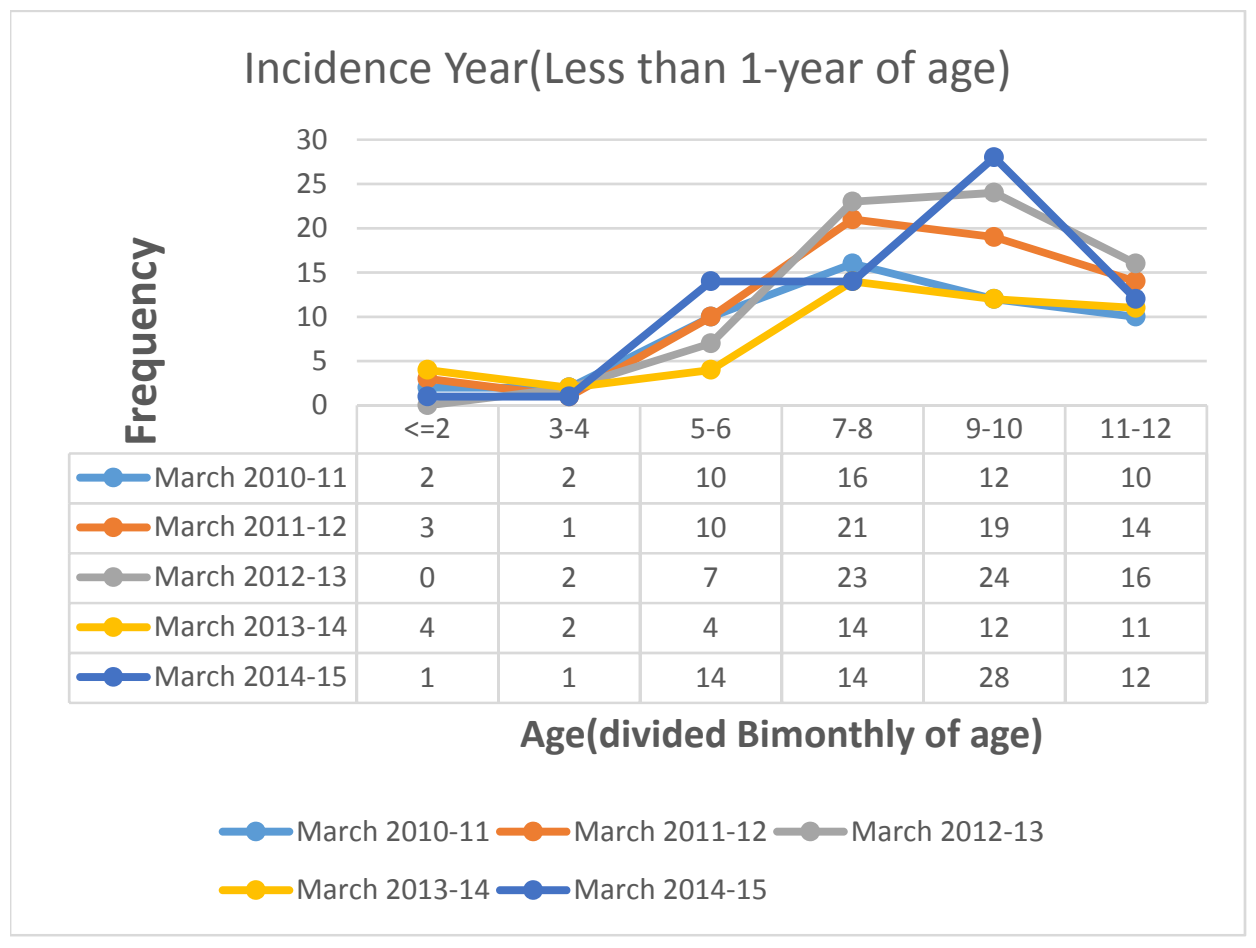

Graph 1. Frequency of under one-year-old infants (divided bimonthly of age) from March 2010 to March 2015 


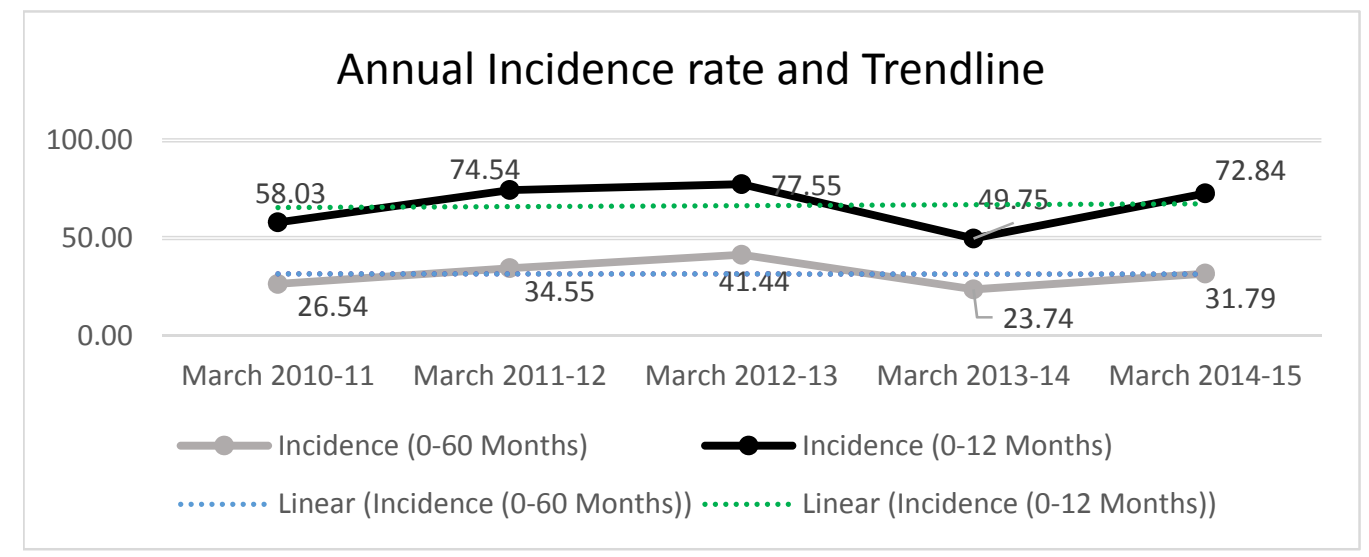

Graph 2. The Annual incidence rate and trend line from March 2010 to March 2015 (Per 100,000 persons)

Table 1. Demographic, clinical, diagnostic, and surgical characteristics of studied patients with intussusception, Tehran, Iran (March 2010-march 2015)

\begin{tabular}{|c|c|c|}
\hline characteristics & & Percentage \\
\hline \multirow[t]{4}{*}{ Season of occurrence } & Spring & 32.3 \\
\hline & Summer & 23.6 \\
\hline & Autumn & 26.6 \\
\hline & Winter & 17.5 \\
\hline \multirow[t]{6}{*}{ Symptoms } & Abdominal pain/Irritability & 94.2 \\
\hline & Nausea and vomiting [Containing biliary vomiting (3.4\%)] & 66.8 \\
\hline & Diarrhea & 38.6 \\
\hline & Fever & 38.6 \\
\hline & Bloody stool including bloody diarrhea and rectorrhagia & 26.1 \\
\hline & GI bleeding & 3.8 \\
\hline \multirow[t]{5}{*}{ Signs } & Tenderness & 24.2 \\
\hline & Abdominal distention & 19.6 \\
\hline & Abdominal mass & 14.1 \\
\hline & Currant jelly stool & 13.6 \\
\hline & Guarding & 8.9 \\
\hline \multirow[t]{6}{*}{ Etiology } & Idiopathic & 47.8 \\
\hline & Mesenteric LAP & 27.7 \\
\hline & Viral respiratory infection & 9.1 \\
\hline & Mesenteric LAP and Viral respiratory infection & 6.6 \\
\hline & Meckel's diverticulum & 2.1 \\
\hline & Other causes (e.g. tumor, history of GI surgery) & 6.7 \\
\hline \multirow[t]{10}{*}{ Diagnostic tool } & US & 75.9 \\
\hline & US and $\mathrm{x}$-ray & 10 \\
\hline & US and contrast enema & 7.4 \\
\hline & Physical examination & 2.5 \\
\hline & Contrast enema & 2 \\
\hline & During surgery & 1.2 \\
\hline & US, X-ray and Contrast enema & 0.5 \\
\hline & Abdominal x-ray & 0.3 \\
\hline & $\mathrm{X}$-ray and contrast enema & 0.1 \\
\hline & Contrast enema & 59.4 \\
\hline Treatment & reduction surgery without resection & 30.3 \\
\hline \multirow[t]{3}{*}{ (722 cases) } & reduction surgery with resection & 3 \\
\hline & Pneumatic enema & 0.4 \\
\hline & Spontaneously & 6.8 \\
\hline \multirow{4}{*}{$\begin{array}{l}\text { Location of the intussusception } \\
\text { (198 cases based on surgery } \\
\text { report) }\end{array}$} & Ileocolic & 87.87 \\
\hline & Ileoileal & 6.06 \\
\hline & Colocolic & 3.02 \\
\hline & Jejunojejunal & 3.02 \\
\hline
\end{tabular}

US= Ultrasonography;LAP=lymphadenopathy

The etiology of intussusception was not found in $47.8 \%$ of the cases, while mesenteric lymphadenopathy was the most common cause of intussusception in patients with a known cause. Other causes including Meckel diverticulum, gastroenteritis, tumor, and history of surgery on gastrointestinal tract were reported in $8.8 \%$ of the patients.

In this survey, $75.9 \%$ of the cases were diagnosed by ul- trasonography. In $1.2 \%$ of the cases, the diagnosis was determined during surgery while the pre-operative diagnosis was not intussusception. The rate of combination of diagnostic tools used in the diagnosis of intussusception is shown in the Table 1 . In $2.5 \%$ of the cases the diagnosis was only performed by physical examination, without any other diagnostic tool. 
From 759 patients, the anatomic location was distinct in 367 cases. The most frequent anatomic location was ileocolic, $(87.87 \%)$ in which ileocecal region was the most frequent site of intussusception $(57.49 \%)$

The type of treatment was not defined in 37 patients' records. Out of 722 remaining cases, $59.4 \%$ of the patients (429 cases) were treated by contrast enema. Other cases were treated by surgery (reduction surgery with and without resection), and pneumatic enema. In addition, $6.8 \%$ of the patients ( 49 cases) recovered spontaneously. Totally, 111 cases were found with failed barium enema that finally underwent surgery.

The mean duration of the hospital stay was $3.53 \pm 2.35$ days $(5.62 \pm 2.26$ and $3.13 \pm 2.10$ days in the groups treated by surgery and non-surgery methods, respectively) with a median of 3 days (from less than 24 hours to 10 days).

The time between presenting symptoms and definite diagnosis was not recorded in 24 patients' files. From 735 remaining patients, $43 \%$ of them were diagnosed in less than 24 hours from the onset of symptoms. No deaths due to invagination or subsequent complications were identified during this 5-year study.

\section{Discussion}

In terms of study duration and the sample size, this research is currently the most comprehensive study on pediatric intussusception in Iran and neighboring countries (1, 17-19).

Regarding the results of this study, the annual incidence of intussusception was 66.54 cases per 100,000 children less than 12 months of age. According to a literature review in childhood intussusception published in 2013, the mean incidence of intussusception was 74 (range: 9-328) per 100, 000 among children younger than 12 months of age (1). The highest and lowest rate of intussusception in Asian countries belonged to South Korea with 328 per 100,000 and Bangladesh with 9 per 100,000 infants less than 12 months of age, respectively. Among Asian and African countries, the results of our study were comparable with Singapore (60 per 100, 000), and Tunisia (62 per $100,000)$ studies $(20,21)$.

We did not find the national incidence of intussusception in neighboring countries to compare our results with.

In this research, the incidence of the disease was highest in the spring which is compatible with the results of another study from northern Iran (17). It might be related to seasonal variations in the incidence of infections especially viral infections as a predisposing factor for intussusception. A study from India showed that the highest number of cases of intussusception occurred in the spring which was similar to the results of our study (22).

Conversely, there are some studies, not only from the neighboring and Asian countries but also from other continents that show no seasonality for the intussusception (1). So, it seems that more investigations are required to explain this contradiction.

Clinical signs and symptoms in the present study were close to those reported in other studies. The most frequent symptom was abdominal pain or irritability, depending on the age of the patients to manifest their complaint. This result was in concordance with the results of an Australian study (23) that abdominal pain was reported in $100 \%$ of their cases. The studies from Korea, India, and Thailand revealed recurrent vomiting, being the most prevalent symptom $(22,24,25)$. It must be considered that the irritability is a subjective finding; also, it is intermittent in this disease, so it is a challenging task to find and record this symptom, especially in retrospective studies, which might be dependent to the time of the first examination. Intussusception can present with a variety of symptoms, and a low prevalence of the 'classical triad' findings, therefore, clinicians should always carry a high index of suspicion. It seems that country-to-country variation in frequency of presenting symptoms of invagination is wide, so the clinicians should keep this point in mind when approaching the patients.

The median time of hospitalization was three days that was less than the results of a study from Thailand (4 days) (25). The time between the disease onset and the hospital admission, is probably a marker for the late diagnosis, increased risk of surgery and related morbidity/mortality.

The male predominance was observed not only in our study but also in all previous studies, although the etiology of this predominance is not well defined. Anyhow, it seems that this predominance was age -dependent and the male predominance was decreased with increasing age in our surveillance.

In this analysis, the classic triad of invagination was reported in $13.7 \%$ of the cases which was lower than the report of a study conducted in India (18.7\%) (22) and higher than the results of the Korean study (408 cases, $8 \%$ ) (24). The clinicians should be advised that the classic triad of invagination does not exist in most of the time and the diagnosis of intussusception should not be only based on these findings.

In this surveillance, the peak incidence of intussusception was between 9 and 10 months of age. The studies from neighboring countries (Pakistan and Iraq) revealed that the peak incidence age was $6-12$ months $(18,19)$ while it was reported 6 months and 5-6 months in Thailand and Zambia; respectively $(25,26)$. According to the results of a literature review performed in 2013, the peak incidence of intussusception was among infants 5-7 months of age (1). It seems that the peak incidence age of intussusception in our study was higher than several other populations but more compatible with the study results of neighboring countries.

The mortality rate was zero in our study while intussusception could be potentially fatal, as the case fatality rate (CFR) was very high (33.7\%) in Zambia (26).

This study has some limitations: The calculated incidence of the disease cannot exactly be generalized to the whole country of Iran. So, it is proposed to perform a large-scale study including different provinces of Iran so that the results of this study can be generalized to the entire population of the country.

Despite extreme effort, there might be an underestimation of real incidence secondary to some reasons such as probable spontaneous reduction of intussusception in some patients before diagnosis. Moreover, there might be 
some cases that have not been included in our study because of the failure to update the final diagnostic code or diagnostic log entry error. Use of patients' data is possible when this information is properly organized and classified. Despite dramatic improvement in this field in recent years, based on studies in this area (27), the coding of diseases in medical recording system of Iran might be suboptimal in some evaluated hospitals.

In this study, patients were identified based on the code of registration in medical records of Tehran hospitals. So it is mentioned as a limitation in the research in order to make it clear to the readers that the authors have taken note of this, and have taken it into consideration.

\section{Conclusion}

These baseline statistics of intussusception are now available to be compared with the imminent postintroduction information to help the vaccine-safety department of Iran in more precise decision-making.

\section{Acknowledgments}

This research was supported and granted by Vice Chancellor of Research, Faculty of Medicine, Iran University of Medical Sciences (IUMS), Tehran, Iran. (Project Code: 9221216224). Also, we wish to acknowledge the help provided by Student Research Committee (SRC), Iran University of Medical Sciences (IUMS), Tehran, Iran. We are also grateful to Zeynab Noorimotlagh for assistance with data gathering, and Ali Mohammad Rousta who moderated this paper and, in that line, improved the manuscript significantly.

\section{Conflict of Interests}

The funding organization had no participation in the study design, data collection, analysis and interpretation, or in manuscript writing. Authors declare no potential conflicts of interests with respect to the research, authorship, and/or publication of this article.

\section{References}

1. Jiang J, Jiang B, Parashar U, Nguyen T, Bines J, Patel MM. Childhood intussusception: a literature review. PloS One. 2013;8(7):e68482.

2. Wong CW, Chan IH, Chung PH, Lan LC, Lam WW, Wong KK, et al. Childhood intussusception: 17-year experience at a tertiary referral centre in Hong Kong. Hong Kong Med J. 2015;21(6):518-523.

3. Jenke AC, Klaaßen-Mielke Y, Zilbauer M, Heininger U, Trampisch H, Wirth S. Intussusception: Incidence and Treatment-Insights From the Nationwide German Surveillance. J Pediatr Gastroenterol Nutr. 2011;52(4):446-451.

4. Buttery JP, Danchin MH, Lee KJ, Carlin JB, McIntyre PB, Elliott EJ, et al. PAEDS/APSU Study Group. Intussusception following rotavirus vaccine administration: post-marketing surveillance in the National Immunization Program in Australia. Vaccine. 2011;29(16):3061-3066.

5. Minney-Smith CA, Levya A, Hodgea M, Jacoby P, Williams SH, Carcione D, et al. Intussusception is associated with the detection of adenovirus $C$, enterovirus $B$ and rotavirus in a rotavirus vaccinated population. J Clin Virol. 2014;61(4):579-584.

6. Lin LH. Perspectives on intussusception. Pediatr Neonatol. 2013;54(3):143-144.

7. Okimoto S, Hyodo S, Yamamoto M, Nakamura K, Kobayashi M. Association of viral isolates from stool samples with intussusception in children. Int J Infect Dis. 2011;15(9):e641-645.

8. Ngendahayo E, Bonane A, Ntakiyiruta G, Munyanshongore A, Mu- ganga N, Bikoroti J, et al. Preparing for safety monitoring after rotavirus vaccine implementation: a retrospective review of intussusception cases among children at a large teaching hospital in Rwanda, 2009-2012. Pediatr Infect Dis J. 2014;33 Suppl 1:S99-S103.

9. Bradshaw CJ, Johnson PRV. Intussusception. Surgery. 2016;34(5):236-240.

10. Chien M, Willyerd F A, Mandeville K, Hostetler MA, Bulloch B. Management of the child after enema reduced intussuseption: hospital or home? J Emerg Med. 2013;44(1):53-57.

11. Yung CF, Chan SP, Soh S, Tan A, Thoon KC. Intussusception and Monovalent Rotavirus Vaccination in Singapore: Self-Controlled Case Series and Risk-Benefit Study. J Pediatr. 2015;167(1):163-168.e1.

12. Baggs J, Haber P. Continued surveillance for intussusception following RotaTeq in VAERS and VSD. In: Advisory Committee on Immunization Practices Meeting. 2010.

13. Mousavi Jarrahi Y, Zahraei SM, Sadigh N, Esmaeelpoor Langeroudy K, Khodadost M, Ranjbaran M, et al. The cost effectiveness of rotavirus vaccination in Iran. Hum Vaccin Immunother. 2016;12(3):794800 .

14. Dutta AK. Rotavirus Vaccination in India - Need for Surveillance of Intussusception. Indian J Pediatr. 2017;84(2):95-96.

15. Bines JE, Kohl KS, Forster J, Zanardi LR, Davis RL, Hansen J, et al. Brighton Collaboration Intussusception Working Group. Acute intussusception in infants and children as an adverse event following immunization: case definition and guidelines of data collection, analysis, and presentation. Vaccine. 2004;22(5-6):569-74

16. Statistical Center of Iran. National Population and Housing Census, 2011: Statistical Center of Iran; 2012 (Available from: https://nnt.sci.org.ir/sites/nnt/SitePages/report_90/ostani/ostani_popula tion report final permision.aspx.)

17. Esmaeili-Dooki MR, Moslemi L, Hadipoor A, Osia S, Fatemi SA. Pediatric Intussusception in Northern Iran: Comparison of Recurrent With Non-Recurrent Cases. Iran J Pediatr. 2016;26(2):e3898.

18. Yousafzai MT, Thobani R, Qazi SH, Saddal N, Yen C, Aliabadi N, et al. Intussusception among children less than 2years of age: Findings from pre-vaccine introduction surveillance in Pakistan. Vaccine. 2018;36(51):7775-7779.

19. Al-Ani MMM, Ghani SH, Maklef OK. Evaluation of Intussusception in Children: Our Experience in 47 Cases. Iraqi J Med Sci. 2017;15(3):250-561.

20. Boudville IC, Phua KB, Quak SH, Lee BW, Han HH, Verstraeten T, et al. The epidemiology of paediatric intussusception in Singapore: 1997 to 2004. Ann Acad Med Singapore. 2006;35(10):674-649.

21. Chouikha A, Fodha I, Maazoun K, Ben Brahim M, Hidouri S, Nouri A, et al. Rotavirus infection and intussusception in Tunisian children: implications for use of attenuated rotavirus vaccines. J Pediatr Surg. 2009;44(11):2133-138.

22. Singh JV, Kamath V, Shetty R, Kumar V, Prasad R, Saluja T, et al. Retrospective surveillance for intussusception in children aged less than five years at two tertiary care centers in India. Vaccine. 2014;32 Suppl 1:A95-98.

23. Justice FA, Auldist AW, Bines JE. Intussusception: trends in clinical presentation and management. J Gastroenterol Hepatol 2006;21(5):842-846.

24. Jo DS, Nyambat B, Kim JS, Jang YT, Ng TL, Bock HL, et al. Population-based incidence and burden of childhood intussusception in Jeonbuk Province, South Korea. Int J Infect Dis. 2009;13(6):e383-388.

25. Khumjui C, Doung-ngern P, Sermgew T, Smitsuwan P, Jiraphongsa C. Incidence of intussusception among children $0-5$ years of age in Thailand, 2001-2006. Vaccine. 2009;27S:F116-F119.

26. Mpabalwani EM, Chitambala P, Chibumbya JN, Matapo B, Mutambo H, Mwenda JM, et al. Intussusception incidence rates in 9 Zambian hospitals, 2007-2011: prerotavirus vaccine introduction. Pediatr Infect Dis J. 2014;33 Suppl 1:S94-98.

27. Jahanbakhsh M, Sharifi M, Ayat M. The status of hospital information systems in Iranian hospitals. Acta Inform Med. 2014 Aug;22(4):268-75. 